\section{Cancer-associated serum antigen}

S. Holdenrieder ${ }^{1}$ und P. Stieber ${ }^{2}$

${ }^{1}$ Institut für Laboratoriumsmedizin, Deutsches Herzzentrum München, Klinik an der Technischen Universität München, München, Deutschland

${ }^{2}$ Institut für Laboratoriumsmedizin, Klinikum der Universität München (LMU), München, Deutschland

\section{Synonym(e) CASA}

Englischer Begriff cancer-associated serum antigen

Definition CASA wurde durch einen monoklonalen $>$ Antikörper definiert, der ein Epitop eines polymorphen epithelialen Muzins erkannte.

Untersuchungsmaterial - Entnahmebedingungen Serum, Plasma.

Analytik $>$ Enzymimmunoassay (EIA), $>$ Radioimmunoassay (RIA), > Immunradiometrischer Assay (IRMA).

Referenzbereich - Erwachsene $<4 \mathrm{U} / \mathrm{mL}$ (methodenabhängig).
Indikation Therapiekontrolle und Nachsorge beim Ovarialkarzinom fraglich.

Interpretation Zwar wurde CASA als sensitiver Marker beim Ovarialkarzinom beschrieben, doch konnte dies verglichen mit CA 125 ( $\triangleright$ Carbohydrate antigen 125) zu keiner nennenswerten Verbesserung der Diagnose führen. Als Anwendungsgebiet kommt eventuell die Verlaufskontrolle vor und nach Therapie beim Ovarialkarzinom infrage, jedoch konnte auch hier bisher keine wesentliche Verbesserung zu CA 125 gezeigt werden.

Diagnostische Wertigkeit Ovarialkarzinom: Therapiemonitoring, Rezidiverkennung fraglich. CASA ist erhöht in Personen höheren Alters, in Rauchern, während der Gravidität im letzten Trimenon sowie in Personen mit malignen Erkrankungen. Insbesondere wird CASA von allen histologischen Arten des Ovarialkarzinoms exprimiert.

\section{Literatur}

Diamandis E, Fritsche HA, Lilja H et al (2002) Tumor markers. Physiology, pathobiology, technology, and clinical applications, 1. Aufl. AACC Press, Washington, DC

Sturgeon CM, Duffy MJ, Stenman UH et al (2008) National Academy of Clinical Biochemistry laboratory medicine practice guidelines for use of tumor markers in testicular, prostate, colorectal, breast, and ovarian cancers. Clin Chem 54:e11-e79 